\title{
KaVA Large Proposal for High-Mass Star-Formation Studies with Multiple Masers
}

\author{
Tomoya Hirota*† \\ National Astronomical Observatory of Japan (NAOJ) and SOKENDAI, Mitaka-shi, Tokyo \\ 181-8588, Japan \\ E-mail: tomova.hirotaenao.ac.ip
}

\section{Kee-Tae Kim $\ddagger$}

Korea Astronomy and Space Science Institute (KASI), Daejeon, 34055, Republic of Korea

\begin{abstract}
We have started a systematic observational study of the $22 \mathrm{GHz}$ water masers and $44 \mathrm{GHz}$ class I methanol masers in high-mass star-forming regions as a four-year KaVA large program since 2016. The primary aim of our project is to investigate dynamical evolution of high-mass young stellar objects and their circumstellar structures by observing spatial distributions and 3dimensional velocity fields of water and methanol maser features. In 2016, we carried out imaging survey of the 25 water masers at $22 \mathrm{GHz}$ and 19 class I methanol masers at $44 \mathrm{GHz}$ selected from our initial source catalog. Based on these results, we have started proper motion measurements of 16 selected water maser sources at $22 \mathrm{GHz}$ in 2018. In addition, we also selected 3 class I methanol maser sources to measure the proper motions for the first time with VLBI. We will present current status of our KaVA large program and future plans.
\end{abstract}

14th European VLBI Network Symposium \& Users Meeting (EVN 2018)

8-11 October 2018

Granada, Spain

\footnotetext{
*Speaker.

${ }^{\dagger}$ Co-PI of KaVA Star Formation Science Working Group in Japan.

${ }^{\ddagger}$ Co-PI of KaVA Star Formation Science Working Group in Korea.
} 


\section{Introduction}

Formation processes of high-mass stars which have $8 M_{\odot}$ or larger are relatively less understood compared with those of low-mass Solar-type star-formation [Ш, []]. Because of shorter evolutionary timescales and smaller population, number of nearby high-mass young stellar objects (HM-YSOs) were limited and hence, statistical studies with sufficiently high resolution were challenging. Recent high resolution and high sensitivity radio interferometer observations (e.g. JVLA, ALMA) allow statistical studies of deeply embedded HM-YSOs to reveal detailed structures of their disk/outflow systems at resolutions better than 0.1 " or an order of 100 au at $1 \mathrm{kpc}$ distance [B]. These observations shed lights on number of unresolved questions regarding high-mass starformation processes, in particular for dynamical properties of HM-YSOs such as mass accretion processes through disk onto HM-YSOs, feedback processes by outflows and/or radiation and origin of high-mass binaries and clusters.

High resolution VLBI observations of HM-YSOs are unique tools to complement interferometer observations. Water $\left(\mathrm{H}_{2} \mathrm{O}\right)$ masers at $22 \mathrm{GHz}$ and class $\mathrm{II}^{1}$ methanol $\left(\mathrm{CH}_{3} \mathrm{OH}\right)$ masers at $6.7 \mathrm{GHz}$ have been employed as representative probes for VLBI observations since they are extremely strong and compact and hence, detectable with VLBI at $\sim 1$ milli-arcsecond (mas) resolution [5, 6, 田]. These maser lines are excellent tracers of shocked gas associated with outflows, circumstellar disks, and expanding ultra-compact HII (UCHII) regions. Multi-epoch maser mapping with VLBI provide 3-dimensional velocity fields including radial velocities and proper motions of compact maser features. This is sometimes essential to estimate inclination angles of disk/outflow systems. Absolute astrometry at 1 mas or better accuracy can locate the dynamical center of HMYSOs.

In order to investigate dynamical structures and their evolution of HM-YSOs and their circumstellar structures, we have initiated a systematic VLBI survey of the multiple maser lines as a large program (LP) of KaVA (KVN and VERA Array), which is a combined array of Korean VLBI Network (KVN) and Japanese VLBI Exploration of Radio Astrometry (VERA). In addition, to the $22 \mathrm{GHz}$ water masers and $6.7 \mathrm{GHz}$ class II methanol masers, we have added the $44 \mathrm{GHz}$ class I methanol maser as a new probe for VLBI observations, which has been detected with KaVA as the first VLBI image [ [ $]$ ]. These multiple masers are complementary with each other for investigating spatial and 3-dimensional velocity structures around HM-YSOs. By combining follow-up observations with VERA for astrometry, Japanese VLBI Network (JVN) and East-Asian VLBI Network (EAVN) for the $6.7 \mathrm{GHz}$ class II methanol masers, Atacama Large Millimeter/Submillimeter Array (ALMA) for thermal molecular lines and continuum, and single-dish telescopes for large-scale structures traced by various molecular lines, we will investigate physical properties and 3-dimensional dynamical structures of disk, outflow, UCHII, and infalling envelope, and their relationship with the evolutionary phases of HM-YSOs [Q].

\section{Observations}

KaVA consists of four $20 \mathrm{~m}$ antennas in VERA and three $21 \mathrm{~m}$ antennas in KVN. The baseline lengths range from 305 to $2270 \mathrm{~km}$, providing the spatial resolutions of 1.2 mas and 0.6 mas at

\footnotetext{
${ }^{1}$ Methanol masers are divided into two classes, class I and class II [ [⿴囗十).
} 
$22 \mathrm{GHz}$ and $44 \mathrm{GHz}$, respectively. The KaVA LP for high-mass star-formation studies have been started since late 2016 for a four-years-long project. For statistical studies, we first selected 87 HM-YSOs in various evolutionary phases, based on the catalogues and single-dish surveys of the $22 \mathrm{GHz}$ water masers, $44 \mathrm{GHz}$ class I methanol masers, and $6.7 \mathrm{GHz}$ class II methanol masers. Most of them are associated with multiple maser species and hence, suitable for our VLBI survey. In the first year, we selected 25 water maser sources at $22 \mathrm{GHz}$ and 19 class I methanol maser sources at $44 \mathrm{GHz}$, for which VLBI data were not available in 2016, to check detectability with KaVA and variability of maser features. Two sources were observed in one track of 8-hour VLBI observations in the first year imaging survey. The recording rate was $1 \mathrm{Gbps}$ with the $16 \times 16 \mathrm{MHz}$ total bandwidths. The velocity resolution and total velocity coverage for the $22 \mathrm{GHz}$ water masers were $0.21 \mathrm{~km} \mathrm{~s}^{-1}$ and $216 \mathrm{~km} \mathrm{~s}^{-1}$, respectively. The correlation processing were done by using the Korea-Japan Joint VLBI Correlator (KJJVC) installed at KASI, Daejeon.

As a result of the first year observations, we selected 16 water maser sources at $22 \mathrm{GHz}$ to conduct proper motion measurements in the second year. The VLBI monitoring in the second year have been started since early 2018 with 2 -month interval and 4 epochs for each source ( $\sim$ half-year or longer monitoring period). Four sources are observed in one track of 8-hour VLBI session by switching target sources every 10 minutes. For some of high-velocity water maser sources, we employed spectral setting of $8 \times 32 \mathrm{MHz}$ total bandwidths to cover velocity widths of $430 \mathrm{~km} \mathrm{~s}^{-1}$ but with coarser velocity resolution of $0.42 \mathrm{~km} \mathrm{~s}^{-1}$. In addition, we also selected 3 class I methanol maser sources at $44 \mathrm{GHz}$ to measure the proper motions for the first time with VLBI. The monitoring observations are still on-going and they will be finished by June 2019.

\section{Results and discussion}

In this section, we will mainly summarize the $22 \mathrm{GHz}$ water maser observations. Figure $\square$ shows examples of spatial distribution maps cross-power spectra of the $22 \mathrm{GHz}$ water masers associated with HM-YSO samples. The spectral profiles for some of our target sources suggest high-velocity components up to $100 \mathrm{~km} \mathrm{~s}^{-1}$ at maximum with respect to their systemic velocities. Thus, these high-velocity water maser sources are most likely tracing outflows driven by HMYSOs. Except for a few examples, most of the observed HM-YSOs show multiple maser features distributed in various size scales ranging from $100 \mathrm{AU}$ to $10000 \mathrm{AU}$. They also have diversity in morphology such as linearly elongated structures, compact shell-like structures, and more complex distributions, suggesting variety of outflow structures. At this moment, we have not yet estimated dynamical properties traced by the water masers because proper motion measurements have not been completed yet.

To understand basic properties of HM-YSOs associated with masers, thermal dust emission and molecular lines are essential. Follow-up ALMA observations at sub-arcsecond resolutions will be compared with our maser maps. We have obtained molecular line data of $\mathrm{SiO}$ and methanol at ALMA band 6 (around 210-230 GHz). These thermal lines are know to be excellent tracers of outflow and shocked gas, and hence, they can fill the gap of maser maps, which prove only spatially sparse structures. Continuum maps provide positions of HM-YSOs and their basic properties based on accurate spectral energy distribution models (SED) [ [W, W]. The 3-dimensional velocity maps of maser features will be essential for constructing accurate SED models by estimating inclination 
angles of disk/outflow systems of HM-YSOs. All these information will be crucial to understanding dynamical evolution of HM-YSOs and maser chronology [Q].
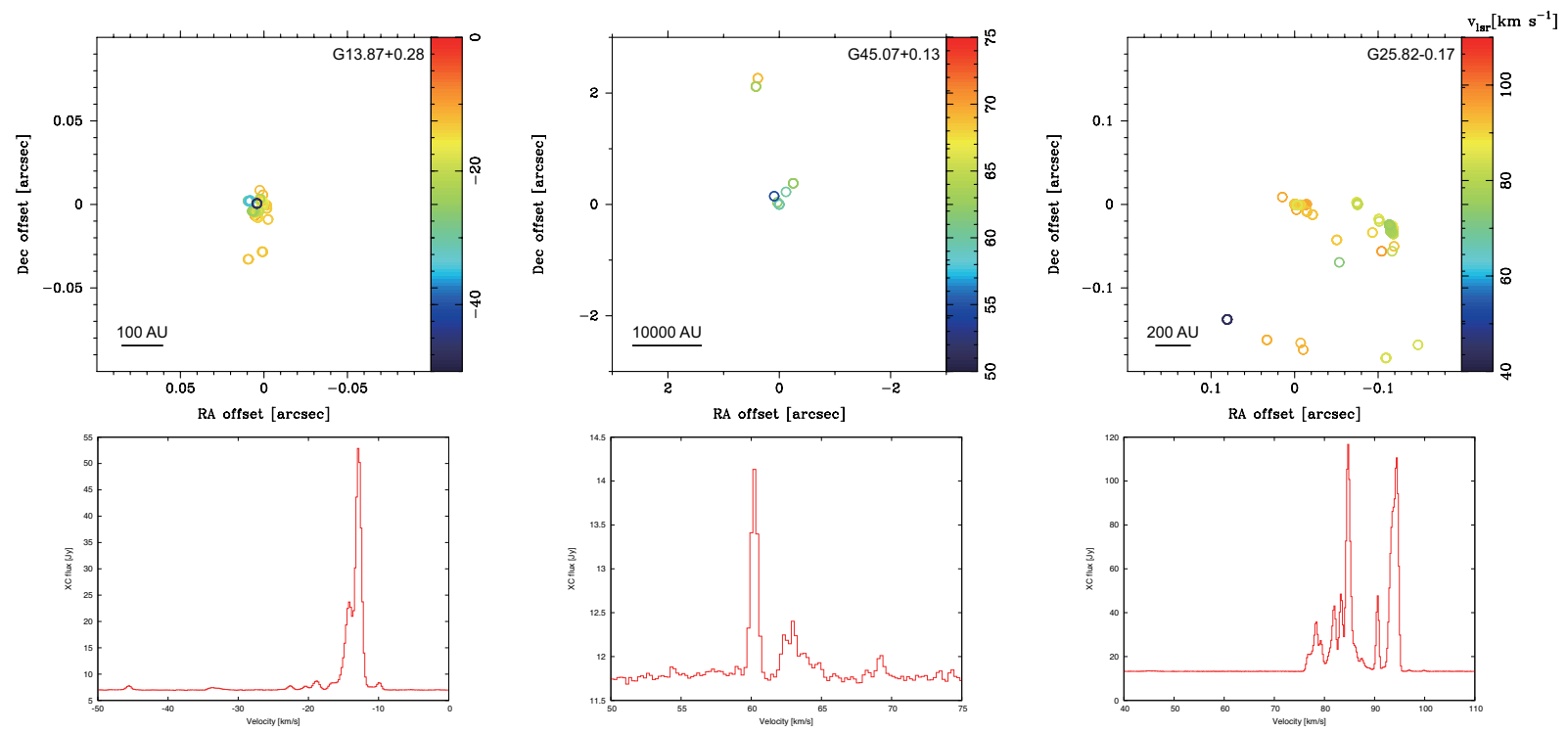

Figure 1: (Top) Examples of spatial distribution maps of the $22 \mathrm{GHz}$ water masers. (Bottom) Example of scalar-averaged cross-power spectra of the $22 \mathrm{GHz}$ water masers.

\section{References}

[1] Zinnecker, H. \& Yorke, H. W., 2007, ARA\&A, 45, 481

[2] Tan, J. C., Beltran, M. T., Caselli, P., et al. 2014, in Protostars and Planets VI, ed. H. Beuther et al. (Tucson, AZ: Univ. Arizona Press), 149

[3] Hirota, T., PKAS, 33, 21

[4] Menten, K. M. 1991, in ASP Conf. Ser. 16, Atoms, Ions, and Molecules: New Results in Spectral Line Astrophysics, ed. A. D. Haschick \& P. T. P. Ho (San Francisco : ASP), 119

[5] Fujisawa, K., Sugiyama, K., Motogi, K., et al. 2014, PASJ, 66, 31

[6] Bartkiewicz, A.,Szymczak, M., \& van Langevelde, H. J. 2016, A\&A, 587, 104

[7] Moscadelli, L., Sanchez-Monge, A., Goddi, C, et al. 2016, A\&A, 585, A71

[8] Matsumoto, N., Hirota, T., Sugiyama, K., et al. 2014, ApJL, 789, L1

[9] Breen, S. L., Ellingsen, S. P., Caswell, J. L., Lewis, B. E. 2010, MNRAS, 401, 2219

[10] Robitaille, T. P., Whitney, B A., Indebetouw, R. et al. 2006, ApJS, 167, 256

[11] Zhang, Y. \& Tan, J. C. 2011, ApJ, 733, 55 